\title{
Tendencia de la producción y el consumo del café en Colombia
}

\author{
Trend in Coffee Production and \\ Consumption in Colombia
}

\author{
Evolução da produção e do consumo de \\ café na Colômbia
}

Olga Lucía Ocampo López * Lina María Álvarez Herrera ${ }^{* *}$

DOI: https://doi.org/10.19053/01203053.v36.n64.2017.5419

Fecha de recepción: 18 septiembre 2016

Fecha de aceptación: 25 de mayo de 2017

\footnotetext{
* PhD. Ingeniería, MSc. Ingeniería, Ingeniera química. Profesora e investigadora asociada Universidad Autónoma de Manizales, Departamento de Mecánica y Producción. Manizales, Colombia. Correo electrónico: olocampo@ autonoma.edu.co, olocampol@unal.edu.co iD http://orcid.org/0000-0002-6394-977X

** Estudiante Administración de Empresas y Negocios Internacionales. Joven investigadora Universidad Autónoma de Manizales. Manizales, Colombia. Correo electónico: lina.alvarezh@autonoma.edu.co (iD http://orcid. org/0000-0003-3471-8961
} 


\section{Resumen}

El objeto de este estudio fue la identificación de tendencias en la producción de café. Se analizaron estadísticas cafeteras en los niveles mundial y nacional, considerando reportes de la Organización Internacional del Café (ICO), el Sistema de Información Agropecuaria y la Federación Nacional de Cafeteros (FNC). Los factores relacionados con la variabilidad climática, el incremento del precio de producción, la escasez de mano de obra y la volatilidad del precio del café afectan la industria cafetera y pueden ser causas estructurales de las tendencias decrecientes en la producción, encontradas en algunos países productores y en algunos departamentos tradicionalmente caficultores de Colombia.

Palabras clave: café, relaciones internacionales, estadísticas cafeteras, agricultura y comercio del café, precio del café.

Clasificación JEL: F14, F53, L66, Q11, Q17. 
Apuntes CENES Volumen 36, Número 64

julio - diciembre 2017. Págs. 139-165

\section{Abstract}

Identifying trends in coffee production was the object of this study that analyzed coffee statistics at global and national level considering reports of the International Coffee Organization (ICO), the Agricultural Information System, and Federación Nacional de Cafeteros de Colombia (the latter is the association of Colombian coffee growers). Factors related to climate variability, increasing of production price, shortages of workforce and volatility of coffee prices, affected the coffee industry and could be structural causes of declining production trends found in some producing countries as well as some departments that traditionally are coffee growers in Colombia.

Keywords: coffee, international relations, coffee statistics, agriculture and coffee trade, coffee price. 


\section{Resumo}

A identificação de tendências na produção de café foi o tema deste estudo, o qual analisou estatísticas de café a nível mundial e nacional, considerando relatórios da Organização Internacional do Café (OIC), o Sistema de Informação Agrícola e a Federação Nacional de Cafeicultores (FNC). Fatores relacionados à variabilidade do clima, ao incremento do preço da produção, à escassez de mão de obra e a volatilidade dos preços do café afetam a indústria do café, estes fatores podem ser causas estruturais do declínio tdas endências de produção encontradas em alguns países produtores e os departamentos tradicionalmente produtores de café na Colômbia.

Palavras chave: café, relações Internacionais, estatísticas do café, agricultura e comércio de café, preço do café. 


\section{INTRODUCCIÓN}

El café es uno de los principales commodities en el ámbito mundial; más del $80 \%$ de la producción se destina al comercio internacional (Quintero \& Rosales, 2014). Como lo indica la Organización Internacional del Café (OIC, 2014a):

El comercio mundial de café es importante para los países exportadores y para los importadores, puesto que generó ingresos de exportación de alrededor de USD 17,9 miles de millones en el año civil 2013 para los países productores y se consumen todos los años alrededor de 600 miles de millones de tazas de café en todo el mundo. (p. 12)
En efecto, es uno de los pilares no solo de la economía colombiana, sino de los países tropicales de América Latina, como lo afirman Quintero y Rosales (2014, p. 2): “el café verde reviste importancia debido a que la producción y el comercio de estos bienes constituyen el pilar de las economías de la mayoría de los países subdesarrollados, principalmente en términos del empleo y de ingresos por exportación".

Las condiciones del mercado, los factores de producción y climáticos influyen sustancialmente en la productividad e inciden en la volatilidad de precios, con consecuencias en los países productores. Según la Organización Internacional del Café (2015): 
La cadena de la oferta se enfrenta con toda una serie de retos. En muchas de las regiones productoras de café los niveles de productividad permanecen dificultados por el bajo nivel de destreza y el acceso limitado a insumo y crédito. Los mercados volátiles sin instrumentos de gestión del riesgo desaniman a efectuar inversión a largo plazo en los cafetales. $(2015$, p. 17)

En Colombia, la industria cafetera tiene gran influencia en la estabilidad económica y social del país (Federación Nacional de Cafeteros de Colombia -FNC-, 2014a). En los últimos años, algunos de los principales departamentos cafeteros presentan transformaciones importantes por los retos que enfrentan no solo por los cambios en el mercado, sino también por situaciones como "el cambio climático, la geografía y los escenarios sociopolíticos locales y territoriales que han evolucionado y definen el entorno para la producción" (FNC, 2014a, p. 13).

Con relación al consumo de café, los mercados desarrollados y los países emergentes representan los mayores niveles (FNC, 2014a, OIC, 2014a). Entre los principales países consumidores se destacan: Estados Unidos con 23 millones de sacos, Brasil con 21 millones de sacos, Alemania con 10 millones y Japón con 7 millones (FNC, 2014a); con consumos inferiores, pero significativos, son relevantes: Finlandia, China, Unión Europea e India (OIC, 2015). La OIC (2015), al evaluar el consumo mundial de café para el período 1990-2012, evidencia un comportamiento estable en los mercados tradicionales que representan más del $50 \%$ del consumo mundial, pero tendencias crecientes en mercados emergentes y en los países exportadores, donde la tasa media de crecimiento anual fue del $2,3 \%$ en los últimos años.

Aunque la Federación Nacional de Cafeteros se ha encargado del desarrollo de alianzas y programas que promuevan el consumo de café, con el objeto de penetrar nuevos mercados, aprovechar las oportunidades que ofrecen los mercados emergentes y mantener el posicionamiento del país como uno de los mayores productor de café en el ámbito internacional (Echavarría, Esguerra, McAllister \& Robayo, 2015; FNC, 2015a), se requieren iniciativas innovadoras que permitan acceder a mercados con valor o a eslabones más avanzados (El País, 2013).

Esguerra y McAllister (2013), en su análisis de tendencias del mercado mundial del café, reportan estos incrementos del consumo mundial y enfatizan sobre los cambios en los factores que influyen en la producción del café. En este sentido, Baker (2010) evaluó el comportamiento en la producción de café con estadísticas históricas desde 1920, con lo cual pudo establecer tres tendencias diferenciadas en el 
comportamiento de la producción en los principales países tradicionalmente productores: a) crecimiento constante; b) volatilidad en la producción pero notable tendencia a la estabilidad y c) tendencias al decrecimiento; indica además que estas tendencias están fuertemente influenciadas por los factores climáticos.

En este panorama es evidente el crecimiento en el consumo y las fluctuaciones en la oferta, con efectos en la volatilidad de los precios del café, que es una característica de la dinámica de este mercado. No obstante, en las últimas décadas es notable el incremento en la vulnerabilidad del caficultor, por efecto del cambio climático (Poveda et al., 2014; Turbay, Nates Jaramillo, Vélez \& Ocampo 2014), lo cual conlleva impactos negativos en los factores de producción, con consecuencias en la productividad.

En este entorno climático cambiante es relevante comprender las tendencias en las estadísticas de producción de café, no solo en el ámbito nacional, como lo reporta la FNC en sus informes de gestión y en su página web, sino también advertir sobre las tendencias en la producción departamental en Colombia, de tal manera que puedan ser consideradas para la toma de decisiones en el sector y en los planes y programas de gestión del riesgo y adaptación.
Este artículo, en primera instancia, realiza un análisis de contexto del mercado mundial y las tendencias de producción, considerando los registros de producción anual reportados por la Organización Internacional del Café, con el fin de brindar una perspectiva global y exponer los factores que influyen en estas tendencias. Finalmente, a partir de las estadísticas del comportamiento de la producción nacional, se identifican las tendencias en la producción y en las áreas cosechadas en los departamentos caficultores colombianos y se analizan algunos factores que influyen en estas tendencias.

\section{METODOLOGÍA}

Este estudio es uno de los productos de una investigación explicativa, que pretende comprender los efectos del cambio y la variabilidad climática en la productividad cafetera (Ocampo, 2017); no obstante, el artículo solamente aborda la identificación de las tendencias en la producción en los países productores y el análisis del comportamiento de las estadísticas cafeteras en los departamentos productores de Colombia. Para su realización se empleó información secundaria que abarcó estadísticas, reportes y artículos científicos relacionados.

Para las estadísticas internacionales se tomó la información de carácter público reportada por la Organización Internacional del Café para el período 
1990-2015, con relación a las estadísticas y las tendencias de la producción total para 53 países productores.

Para Colombia, se analizaron las series de producción, áreas cosechadas y cultivas en 20 departamentos caficultores. La exploración de información secundaria disponible evidencia la ausencia de series históricas de producción departamental, de carácter público, en las estadísticas de la Federación Nacional de Cafeteros y en los reportes de los Comités Departamentales de Cafeteros. Por tanto, se tomó como base la información de producción y áreas cosechadas reportadas por las Evaluaciones Agropecuarias Municipales (EVA) disponibles en el Sistema de Información Agropecuaria para el período 2007-2013 y las estadísticas de la Federación relacionadas con las áreas cultivadas de café para el período 2002-2015.

Para la evaluación de tendencias, se tuvo en cuenta un análisis gráfico efectuado en el software Microsoft Excel; se aplicaron pruebas estadísticas para confirmación de tendencias: crecientes (A), volatilidad con tendencia a la estabilidad (B) y decrecientes (C), como lo reporta Baker (2010). Inicialmente, se determinaron modelos de regresión lineal por técnicas paramétricas y se procedió a la verificación de los supuestos, teniendo en cuenta análisis de significancia de los $\beta$ individuales $(\alpha<0,10)$, la prueba global del modelo $(\alpha<0,10)$; distribución normal de los errores (Shapiro-Wilk), homocedasticidad (prueba de White) y no correlación en los errores (Durbin Watson); para tal efecto se empleó el software estadístico SSPS. No obstante, dado que no se cumplieron los supuestos en todas las series, se seleccionaron pruebas clásicas, no paramétricas, para el análisis de tendencias como el test de Mann-Kendall (Hirsch, Slack \& Smith, 1982); se empleó además el coeficiente de correlación de Spearman, para confirmar los niveles de correlación de las series de tiempo, con una significancia del $90 \%$; el software IBM SPSS fue utilizado para estas pruebas.

A continuación se describen los resultados obtenidos de la revisión de información secundaria y la identificación de tendencias en el mercado global y nacional.

\section{ANÁLISIS DE ESTADÍSTICAS CAFETERAS Y TENDENCIAS EN EL MERCADO GLOBAL}

En los últimos 60 años, el consumo mundial de café se incrementó a una tasa media de crecimiento anual del 2,6\%, pasando de 57,9 millones de sacos en 1964 a 150,3 millones en el 2014 (OIC, 2014b; OIC, 2016). Para el año 2015, el consumo estimado de 152,1 millones de sacos sugiere un incremento de 1,2\% (OIC, 2016). Las proyecciones futuras estiman un alza 
sostenida hasta el 2020, donde se espera un consumo de 175 millones de sacos de $60 \mathrm{~kg}$; este crecimiento será impulsado por el incremento del consumo en los mercados no tradicionales como China, Corea del Sur y Rusia (La República, 2014).

Sin embargo, no se evidencian los mismos niveles de crecimiento en la producción del grano. Según la Figura 1 , que compila las estadísticas de producción reportadas por la International Coffee Organization -ICO- (2016b) para el período 1990-2015, la tasa de crecimiento promedio anual para este período ha sido del $2,2 \%$, pero en lo últimos años se ha presentado desaceleración; la mayor producción se registró en el año cafetero 2012-2013 con 147,95 millones de sacos; para los años subsiguientes se tienen reducciones hasta del $4,4 \%$ en el período 2014/2015.

Para el período 1970-2013, Suramérica, Asia y Oceanía registraron un crecimiento constante en la producción del grano; Centroamérica presentó un comportamiento estable y África evidenció tendencias descendientes (OIC, 2014b). El anuario de la OIC (2015) presenta las estadísticas de producción de café por continente para el período 2014-2015: África 16,6 millones de sacos; Asia y Oceanía 45,7 millones de sacos; Centroamérica 18,8 millones de sacos y Suramérica 67,2 millones de sacos; las fluctuaciones con relación a la cosecha 2013-2014 fueron $+2,5 \%$ en África, $-1,9 \%$ en Asia y Oceanía, $+7.1 \%$ en Centroamérica y $-6,3 \%$ en Suramérica (OIC, 2015).

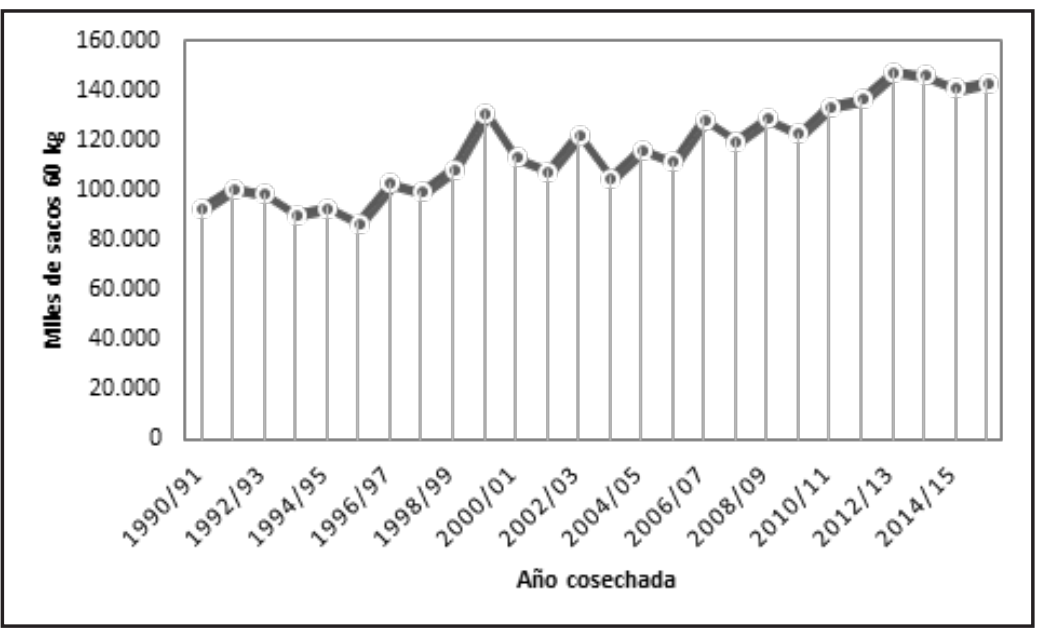

Figura 1. Estadísticas de producción de café en el ámbito global 1990-2016.

Fuente: (ICO, 2016b) 
Los principales países productores y su participación porcentual para el año 2015, según el análisis de las estadísticas históricas de la ICO (2016b), son los siguientes: Brasil $(30,2 \%)$, Vietnam (19,2\%), Colombia (9,4\%) e Indonesia $(7,7 \%)$. Con relación a las exportaciones de café, el análisis de las estadísticas de la ICO (2016a) para el período 1990-2015, evidencia tendencias crecientes. Los principales exportadores en el 2015 fueron: Vietnam $(25,2 \%)$, Brasil $(22,2 \%)$, Colombia $(11,9 \%)$ e Indonesia $(6,8 \%)$, (ICO, 2016a).

Baker (2010) analizó las estadísticas cafeteras para el registro histórico 1920-2010 e identificó tres tendencias que exhiben comportamiento no lineal: la primera (A) identifica tendencia al crecimiento en la producción durante el periodo evaluado, y se presenta en países como Vietnam, Indonesia, Etiopia y Perú. La segunda (B) representa la volatilidad en la producción, pero con una tendencia a mantener los niveles de producción, y se da en países como Colombia, Uganda, México y Venezuela. En la tercera (C) se demuestra un pico en la producción y luego una tendencia a la reducción, como es el caso de Kenia, Ecuador, Camerún y el Salvador.

Tomando como base la clasificación de tendencias propuesta por Baker (2010), se analizaron las estadísticas de 53 países productores y exportadores de café, reportados por la ICO (2016b) para el período 1990-2015. Los resultados del análisis se compilan en la Tabla 1, donde se identifican los tres tipos de tendencias. Considerando la clasificación de los países por el tipo de café predominante en el país y su calidad (ICO, 2016b) se muestran las siguientes tendencias en la producción: i) arábicas suaves colombianos: volatilidad en Colombia y Tanzania; pero decrecientes en Kenia. ii) Otros arábicas suaves: crecimiento en Honduras, India, Nicaragua y Perú; volatilidad en Bolivia, Guatemala, Papúa Nueva Guinea, Venezuela y Zambia, y reducciones en los demás países.

iii) Arábicas naturales brasileños y otros arábicas naturales: crecientes en Brasil, Etiopía, Timor Leste y Yemen, pero decrecientes en Paraguay. iv) Robustas: crecientes en Guinea, Indonesia, Liberia, Uganda, Vietnam; volatilidad en Ghana, Sierra Leona y Togo; y reducciones en los demás países. 
Tabla 1. Tendencias en la producción de café para el período 1990-2015

\begin{tabular}{|c|c|c|}
\hline $\begin{array}{l}\text { A: Tendencias } \\
\text { crecientes }\end{array}$ & $\begin{array}{l}\text { B: Volatilidad con tenden- } \\
\text { cia a la estabilidad }\end{array}$ & C: Tendencias decrecientes \\
\hline Brasil & Bolivia & Angola \\
\hline Etiopía & Colombia & Burundi \\
\hline Guinea & Ghana & Camerún \\
\hline Guyana & Guatemala & Congo \\
\hline Honduras & Papúa Nueva Guinea & Costa de Marfil \\
\hline India & Sierra Leona & Costa Rica \\
\hline Indonesia & Tanzania & Cuba \\
\hline Lao & Togo & Ecuador \\
\hline Liberia & Venezuela & El Salvador \\
\hline Nepal & Zambia & Filipinas \\
\hline Nicaragua & & Gabón \\
\hline Perú & & Haití \\
\hline Timor Leste & & Jamaica \\
\hline Uganda & & Kenia \\
\hline Vietnam & & Madagascar \\
\hline \multirow[t]{12}{*}{ Yemen } & & Malawi \\
\hline & & México \\
\hline & & Nigeria \\
\hline & & Panamá \\
\hline & & Paraguay \\
\hline & & República Centroafricana \\
\hline & & República Dominicana \\
\hline & & Ruanda \\
\hline & & Sri Lanka \\
\hline & & Tailandia \\
\hline & & Trinidad y Tobago \\
\hline & & Zimbabue \\
\hline
\end{tabular}

Fuente: elaboración propia a partir de ICO (2016b) 
Para los principales cuatro países pro- productores del grano presentan tenductores de café, el comportamiento dencias crecientes en la producción se ilustra en la Figura 2. Con excep- para el período evaluado 1990-2015. ción de Colombia, estos principales
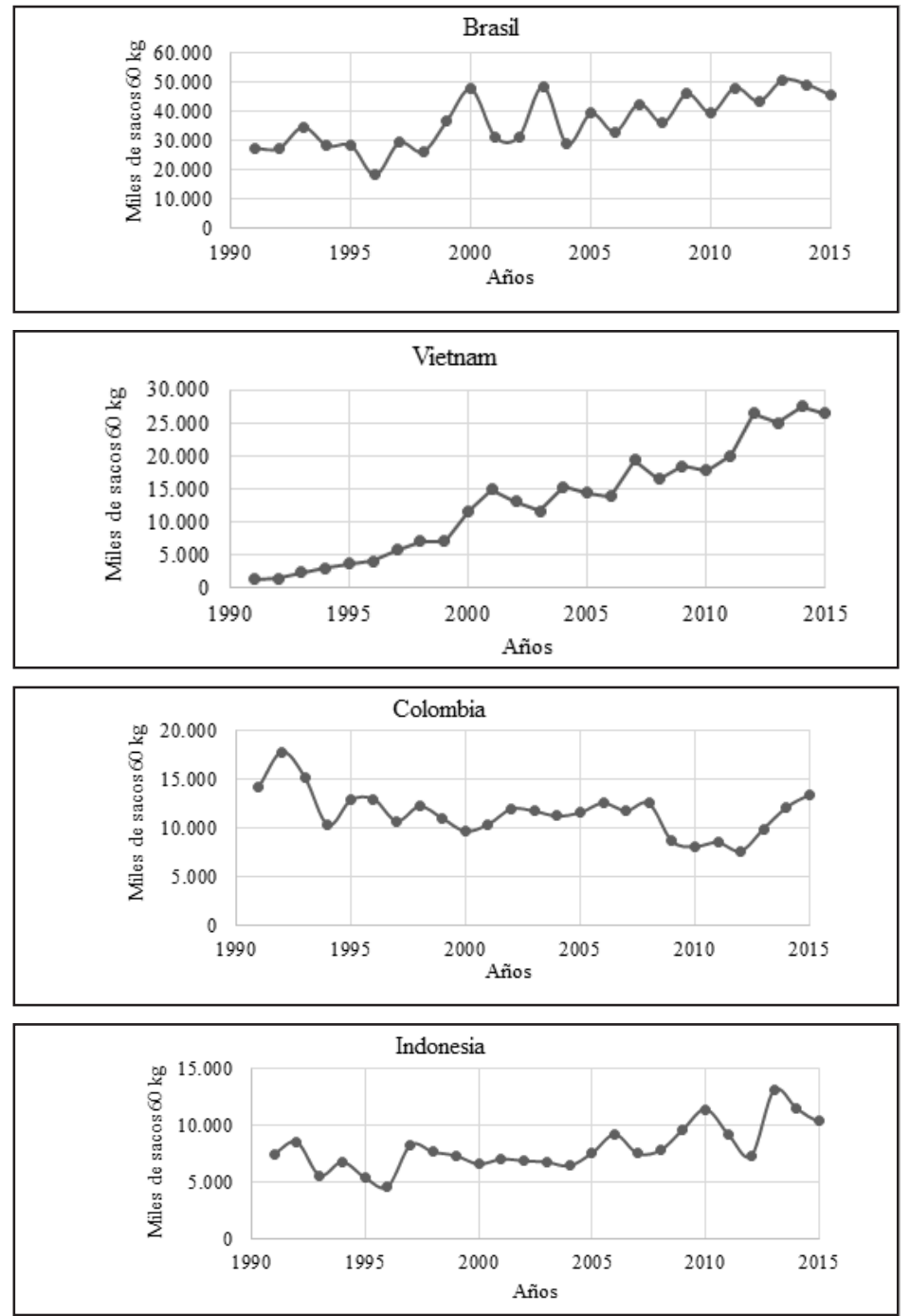

Figura 2. Tendencias en la producción de café 1990-2015 en los principales países productores.

Fuente: elaboración propia a partir de ICO (2016b) 
Las estadísticas de Brasil demuestran un aumento constante en la producción desde 1990-1991, cuando se registraron 27.2 millones de sacos frente a 50,8 millones para el 2012-13, que equivale a un incremento del 53,68 \% en la producción, según el análisis de estadísticas reportadas por ICO (2016b). La Federación Nacional de Cafeteros (2015a) afirma que la producción de Brasil presenta altos niveles de volatilidad, debido a las condiciones climáticas que impactan fuertemente los niveles productivos, ya sea por heladas o sequías o por ataques de la roya. Los factores climáticos, en especial la presencia de heladas en Brasil, han influido sustancialmente en el precio del grano; en el año 2014, por ejemplo "el precio internacional del café presentó un gran nivel de volatilidad, soportado en gran medida en el alto nivel de incertidumbre que generaba la situación climática en Brasil y su impacto en el volumen de producción" (FNC, 2015a, pp. 11-12).

El aumento de temperaturas por efecto del cambio climático se ha convertido en "la amenaza más importante a la producción sostenible de café" (OIC, 2014 a, p. 28). En el mundo, el cambio climático es una amenaza, social y económica, por la posibilidad de impacto en la agricultura, uno de los sectores más vulnerables (Ocampo, 2011).
Entre los efectos del cambio climático se encuentra la exacerbación de los fenómenos de El Niño y La Niña, que modulan la variabilidad climática interanual en los países tropicales (Ocampo, Vélez \& Londoño, 2014; Sauchyn, Upegui, Masiokas, Ocampo, Cara \& Villalba, 2016; Turbay et al., 2014), donde la mayoría de los cultivos, como el café, no están dotados de irrigación y, por tanto, esta variabilidad climática juega un rol importante en la productividad (Poveda et al., 2014); así lo indica la Organización Internacional del Café (OIC, 2016, p. 3), "el tiempo seco causado por El Niño podría afectar la producción de Vietnam, Indonesia y Colombia".

La producción cafetera se ha impactado por la variabilidad climática, pero las plantaciones se han podido recuperar (Tucker, Eakin \& Castellanos, 2010; Poveda et al., 2014). Los impactos futuros del cambio climático podrían ser más severos; en algunos sitios por efectos del incremento de la temperatura (Poveda et al., 2014; Laderach, et al., 2011; Schroth et al., 2009) y por lo cambios en la dinámica de las plagas y enfermedades (CORPOICA, 2011), la caficultura podría ser no viable (Ramírez et al., 2012; Ocampo, 2017).

Estas variaciones en la oferta y demanda traen repercusiones en el precio del grano e influyen sustancialmente en la industria cafetera. Para el 2016, por 
ejemplo, "aumenta el consumo mundial de café, pero los precios siguen bajos... dados los niveles de inventarios en los países importadores y la posibilidad de una gran cosecha en Brasil en 2016/2017" (OIC, 2016, p.1).

En el ámbito global, las tendencias del mercado mundial del café están relacionadas con la volatilidad de los precios y la producción, el incremento de los costos de producción en los principales países productores como Colombia y Brasil, el aumento en la demanda de café -en especial robusta y cafés especiales-, la mayor segmentación de mercados, la concentración de la compra y la comercialización (Echavarría et al., 2015; Esguerra \& McAllister, 2013).

El incremento en la demanda de café variedad robusta influye en la producción de países tradicionalmente productores de café arábiga, como Colombia; así lo indican Echavarría et al. (2015, p. 5): "la demanda mundial por café robusta ha crecido a tasas mucho mayores que la de los cafés arábigas, y los arábigas suaves colombianos han perdido participación frente a otros suaves".
No obstante, los cafés suaves de alta calidad siguen siendo fundamentales en las tendencias del mercado mundial. En Europa, por ejemplo, según The Centre for Promotion of Imports from Developing Countries (CBI, 2016), se presentan diferentes tendencias: 1) "La tercera ola de café" relacionada con la promoción del café como un producto artesanal de alta calidad que impulsa la demanda de cafés especiales. 2) Demanda de café ético que promueve nichos como Fairtrade y orgánico. 3) Innovación que busca mejorar la calidad, reducir los costos ambientales y económicos y adaptar la caficultura al cambio climático.

\section{ANÁLISIS DE ESTADÍSTICAS CAFETERAS Y TENDENCIAS EN EL MERCADO NACIONAL}

Para identificar las tendencias en la producción, se parte del análisis de estadísticas reportadas en las EVA para el período disponible 2007-2013. Los resultados se consolidan en la Tabla 2 , donde se identifican dos tipos de tendencias: estabilidad y decrecimiento. No obstante, se detectan tendencias decrecientes en los departamentos del denominado Eje Cafetero colombiano. 
Tabla 2. Tendencias en la producción departamental de café en Colombia para el período 2007-2013

\begin{tabular}{|c|c|c|}
\hline $\begin{array}{c}\text { A: Tendencias } \\
\text { crecientes }\end{array}$ & $\begin{array}{c}\text { B: Volatilidad con tenden- } \\
\text { cia a la estabilidad }\end{array}$ & C: Tendencias decrecientes \\
\hline & Antioquia & Boyacá \\
\hline & Bolívar & Caldas \\
\hline & Caquetá & Cundinamarca \\
\hline & Cauca & Quindío \\
\hline & Cesar & Risaralda \\
\hline & Chocó & Valle del Cauca \\
\hline & Huila & \\
\hline & La Guajira & \\
\hline & Magdalena & \\
\hline & Meta & \\
\hline & Nariño & \\
\hline & Norte de Santander & \\
\hline & Santander & \\
\hline
\end{tabular}

Fuente: elaboración propia con base en Ministerio de Agricultura y Desarrollo Rural (2014)

Para explicar este comportamiento es necesario evaluar las tendencias en el área cosechada para el período de análisis 2007-2013, considerando la misma fuente de información, Ministerio de Agricultura y Desarrollo Rural (2014). Los resultados confirman las siguientes tendencias: a) crecientes: Caquetá y Meta; b) estabilidad: Bolívar, Casanare, Cauca, Cesar, Chocó, Huila, La Guajira, Magdalena, Nariño, Norte de Santander, Quindío, Santander y Tolima y Valle del Cauca; c) decrecientes: Antioquia, Boyacá, Caldas, Cundinamarca y Risaralda.

En consecuencia, el comportamiento decreciente en la producción en departamentos tradicionalmente caficultores como Caldas y Risaralda, puede explicarse por las tendencias decrecientes en las áreas cultivadas.

Considerando las estadísticas para el período 2007-2013 reportadas por las 
EVA, se analizó la productividad, expresada en peso por hectárea, donde los mayores valores promedio se obtienen en Caldas y los menores en el Valle del Cauca. Se confirman tendencias decrecientes en Boyacá, Huila, Nariño, Quindío, Risaralda, Tolima y Valle del Cauca. Estos resultados pueden explicar las tendencias decrecientes en la producción en departamentos tradicionalmente caficultores como Quindío, Risaralda y Tolima, para el período evaluado.

A partir de las estadísticas de la Federación Nacional de Cafeteros (2016a), en relación con el área cultivada con café total departamental, puede evaluarse la tendencia para un mayor período de registro 2002-2015. Los resultados se resumen en la Tabla 2 , donde se evidencian los tres tipos de tendencias. Aunque el efecto neto es creciente en el nivel nacional, tal como se muestra en la Figura 3, es necesario advertir sobre las tendencias decrecientes en las áreas cultivadas en los departamentos que conforman el denominado "triángulo del café", Caldas, Quindío y Risaralda, y cuya economía está fuertemente ligada por tradición a la producción del grano.

Tabla 2. Tendencias en las áreas cultivadas de café en Colombia 2002-2015

\begin{tabular}{|c|c|c|}
\hline $\begin{array}{c}\text { A: Tendencias } \\
\text { crecientes }\end{array}$ & $\begin{array}{c}\text { B: Volatilidad con tenden- } \\
\text { cia a la estabilidad }\end{array}$ & C: Tendencias decrecientes \\
\hline Antioquia & Boyacá & Caldas \\
\hline Caquetá* $^{\star}$ & Norte de Santander & Chocó $^{*}$ \\
\hline Casanare* $^{\star}$ & & Cundinamarca \\
\hline Cauca & & Risaralío \\
\hline Cesar & & Valle del Cauca \\
\hline Guajira & & \\
\hline Huila & & \\
\hline Magdalena & & \\
\hline Meta & & \\
\hline Nariño & & \\
\hline Santander & & \\
\hline Tolima & & \\
\hline
\end{tabular}

Nota: * período de análisis 2007-2015.

Fuente: elaboración propia a partir de FNC (2016a). 
Según la Superintendencia de Industria y Comercio (SIC, 2012), entre el 2007 y el 2011, los cultivos bajo sombra o sombra parcial, se redujeron 40,38 mil hectáreas en todo el territorio nacional. En algunos departamentos disminuyeron las hectáreas, como en: Quindío (-30,21\%), Cundinamarca $(-20.9 \%)$, Valle $(-12,42 \%)$, Risa- ralda $(-11,48 \%)$, Caldas $(-9,34 \%)$ y Boyacá $(-0,74 \%)$; sin embargo, se presentó incremento en Huila $(65,09 \%)$, Nariño (53,81\%), Guajira $(26,74 \%)$, Cauca (23,58 \%), Magdalena (13,25\%), Cesar (16,73\%), Santander (7,44 \%), Tolima (4,07\%), Norte de Santander $(2,65 \%)$ y Antioquia (2,48 \%), (SIC, 2012).

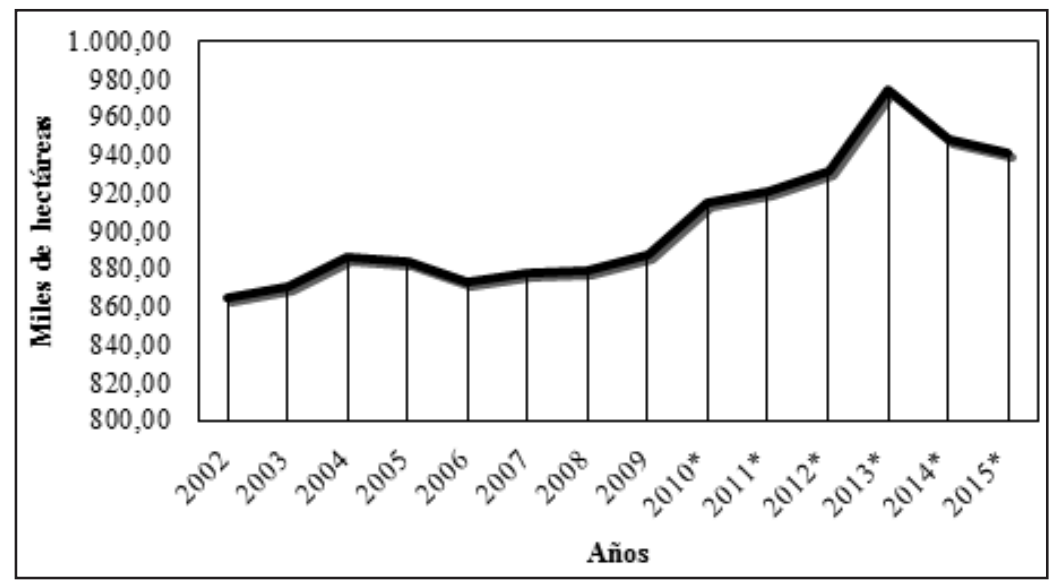

Figura 3. Áreas cultivadas con café en Colombia 2002-2015.

Fuente: FNC (2016a)

Las estadísticas históricas del área cultivada de café por departamento para el período 2002-2015 (FNC, 2016a), registran la mayor área en el año 2013 con 974010 ha; por tanto, se presentaron reducciones del $2,6 \%$ en el 2014 y $3,4 \%$ en el 2015 ; sin embargo, estas fluctuaciones cambian en el nivel departamental. Por ejemplo, para Caldas, la máxima área cultivada para el período de análisis (90870 ha), se registró en 2004, mientras que la mínima (72370 ha), en 2015.
En Colombia, el sector cafetero históricamente ha desempeñado un papel importante en la economía (FNC, 2014a), pues representa el sustento para aproximadamente 560.000 caficultores y sus familias (FNC, 2013); la mayoría hace parte de la Federación, la cual ha brindado asistencia técnica para cumplir con los estándares de calidad requeridos para la exportación del café y garantizar la calidad del grano (FNC, 2015a), dado que el segmento de mercado al que pertenece el 
café colombiano corresponde al tipo de cafés arábicos lavados.

Colombia contribuye con alrededor del $30 \%$ de producción de este tipo de café (Superintendencia de Industria y Comercio -SIC-, 2012), en consecuencia, es el mayor productor de café de calidad y punto de referencia del grano de calidad superior en el mundo (OIC, 2015). Las condiciones ambientales, el sistema de gestión y el respaldo a la calidad hacen parte de los elementos diferenciales del café colombiano (FNC, 2013).

Sin embargo, durante las últimas tres décadas se han presentado dificultades y problemas en la producción (SIC, 2012), entre los cuales se destaca la baja productividad por hectárea. Para el año 2015 fue 16,8 sacos por hectárea, mientras que para Brasil alcanzó 24,5 sacos por hectárea para el mismo periodo (FNC, 2015b). Se evidencia además escasez de mano de obra, especialmente en la recolección de café, que afecta no solo la productividad, sino también deja expuesto el cultivo al ataque de plagas, por la falta de personal para el mantenimiento de las fincas (Portafolio, 2015; La República, 2015). Otros aspectos que afectan la producción del café están relacionados con la implementación de tecnologías en todas las fases de la producción, pues en Colombia se dificulta por las condiciones topográficas y la presencia de múltiples pases por efecto de las condiciones climáticas (Cadena, 2005; Salazar et al., 2016; Ocampo-López et al., 2017).

Los factores que inciden en el sector cafetero están relacionados con la incertidumbre y volatilidad de los precios internacionales del café y del tipo de cambio; el aumento del precio de la mano de obra en 4,6\%, tomando como base el SMMLV ${ }^{1}$ establecido para el 2015; el costo de los fertilizantes, que subió cerca de $12 \%$ en consonancia con la devaluación del peso y los riesgos asociados a la variabilidad climática (FNC, 2015a; FNC, 2015b; Turbay et al., 2014).

No obstante, la relación entre la producción y las exportaciones de café de Colombia se mantuvo con ritmo positivo de crecimiento entre el 2014 y el 2015 (FNC, 2015b), y de esta manera el país recobró la posición que había perdido en los mercados extranjeros, como efecto de la caída de la cosecha en el 2009, cuando se registraron 7,8 millones de sacos (Echavarría et al., 2015).

La producción de café en Colombia para el período 2014-2015, alcanzó 13,6 millones de sacos, que representa un incremento del $12 \%$ con relación al año cafetero anterior, estimada en

\footnotetext{
1 Salarios mínimos mensuales legales vigentes.
} 
12,1 millones de sacos (FNC, 2015b; ICO, 2016b). En el primer trimestre del 2016 se registró una producción alrededor de 3,2 millones de sacos de $60 \mathrm{~kg}$, que representa un aumento del $9 \%$ (FNC, 2016b).

Por su parte, las exportaciones de café alcanzaron un valor total de $2.787 \mathrm{mi}-$ llones de dólares en 2015, superior a los 2.685 millones de dólares registrados en 2014 (FNC, 2015b). Durante el 2015, las ventas externas fueron de $12,5 \mathrm{mi}-$ llones de sacos de 60 kilos, $15 \%$ más en comparación con los 10,9 millones de sacos exportados en el periodo anterior (FNC, 2015b). Los países que importaron los mayores volúmenes de café verde colombiano en el 2015, expresados en miles de sacos de 60 $\mathrm{kg}$ de café verde equivalente, fueron: Estados Unidos con 5289, Japón con 1.330, Alemania con 1.109, y Bélgica con 919 (FNC, 2016c).

No obstante, la Figura 2 exhibe la gran volatilidad en la producción de café en Colombia. Algunos de los factores que más influyen en esta producción son los climáticos y, especialmente, los eventos fríos del fenómeno Oscilación del Sur ENOS (FNC, 2014b; Jarami1lo-Robledo \& Arcila-Pulgarín, 2009). Para el período evaluado se presentaron diferentes eventos del fenómeno de Oscilación del Sur ENOS, según el Índice Oceánico ONI, reportado por la NOAA (2016): 1) La Niña: agosto 2007-junio 2008; julio 2010-febre- ro 2012; 2) El Niño: julio 2009-abril 2010; noviembre 2014-mayo 2016.

El fenómeno de La Niña registrado durante 2010-2011 fue uno de los más fuertes en Colombia (BID-CEPAL, 2012) y produjo notable afectación en los cultivos de café: "impactó la floración de las cerezas del café, así como la sanidad y el crecimiento de los árboles de café" (Poveda et al., 2014, p. 5). Las alteraciones climáticas asociadas con el fenómeno de La Niña afectaron considerablemente la ocurrencia de las floraciones; en consecuencia, la producción de café bajó significativamente, y para el 2011 y el 2012 solo alcanzó 7.8 y 7.7 millo-nes de sacos, respectivamente (FNC, 2011, 2012). "Los costos de producción se incrementaron con la caída en la productividad por hectárea, por el mal estado de las vías y por los altos costos de los fertilizantes" (Turbay et al., 2014, p. 102). Los caficultores se vieron claramente expuestos a "una disminución de sus ingresos y de las condiciones de vida en el medio rural, aunado a otros factores socioeconómicos" (Quintero \& Rosales, 2014, p. 2).

Por su parte, el fenómeno de El Niño 2015-2016 fue uno de los más fuertes registrados tanto en Colombia como en el mundo. Colombia tuvo que invertir 1,6 billones de pesos para prevenir y atender las emergencias producto de la falta de lluvias y la sequía (El Tiempo, 2016a). Para establecer los efectos ad- 
versos del fenómeno de El Niño en la caficultura, la Federación Nacional de Cafeteros hizo un estudio en 7.048 fincas cafeteras, que arrojó los siguientes resultados promedio: $19,4 \%$ de granos flotantes; $33 \%$ de granos inmaduros y $16,5 \%$ de árboles con marchitez; los mayores defectos se detectaron en Antioquia, Caldas, Cundinamarca, Huila, Meta, Risaralda, Valle del Cauca y Tolima (El Espectador, 2016). Las pérdidas estimadas por este fenómeno para el 2016 están alrededor de 0.5 billones para el sector cafetero (El Tiempo, 2016b).

Colombia es vulnerable tanto al cambio como a la variabilidad climática (Ocampo, Vélez \& Londoño, 2014). Los posibles impactos afectan los sectores productivos, como la caficultura, y por tanto, se requiere el desarrollo de estrategias que preparen al país para afrontar los retos que impone el desarrollo económico y social (Departamento Nacional de Planeación -DNP-, 2011). En la caficultura es fundamental adaptar las medidas y estrategias para reducir la vulnerabilidad frente a la variabilidad climática. En eventos cálidos El Niño, el manejo adecuado de arvenses y sombrío es fundamental, al igual que la vigilancia y control en los cafetales ante la broca, el minador de la hoja y la arañita roja. En eventos fríos La Niña, se deben vigilar la dispersión del hongo de la roya, el mal rosado y el ataque de babosas, al igual que los sistemas de drenaje, y atender las recomendaciones técnicas para el trazado geométrico del cultivo, la densidad de siembra y control de arvenses (Poveda et al., 2014; FNC, 2014b).

En respuesta a los impactos del cambio y la variabilidad climáticos, el Plan Nacional de Adaptación al Cambio Climático -PNACC- busca incidir en los procesos de planificación ambiental, territorial y sectorial para que se incluyan las proyecciones climáticas en la toma de decisiones, de tal manera que se busque la reducción de la vulnerabilidad de las poblaciones, ecosistemas y sectores productivos y se incremente la resiliencia para responder a los eventos climáticos (DNP, 2011).

La Federación Nacional de Cafeteros es pionera en el campo y está adelantando ambiciosos programas de renovación con variedades resistentes a la roya y focalizando sus esfuerzos en temas de investigación en nuevas variedades. Sin embargo, la conservación del suelo, el uso adecuado del sombrío, los sistemas de alerta temprana de broca y roya, el conocimiento oportuno del clima en las diferentes regiones y el uso de la tecnología para llevar soluciones prácticas relevantes y oportunas hacen parte de lo que se ha denominado "caficultura climática inteligente" (FNC, 2011, 2012) y que debe ser integrada no solo en el sector cafetero, sino también en las diferentes cadenas productivas. 
Apuntes CENES Volumen 36, Número 64

julio - diciembre 2017. Págs. 139-165

\section{CONCLUSIONES}

El análisis de las estadísticas cafeteras reportadas por la Organización Internacional del Café permite identificar comportamientos diferenciados en los países productores de este grano: 1. Tendencias crecientes en los principales países productores, como Brasil, Vietnam e Indonesia y en algunos países hispanoamericanos, como Honduras, Nicaragua y Perú. 2. Tendencias decrecientes en la mayoría de países africanos y en algunos latinoamericanos, como Costa Rica, Cuba, Ecuador, El Salvador, Haití, Jamaica, México, Panamá, Paraguay y Trinidad y Tobago. 3. Volatilidad con tendencia a la estabilidad en Colombia y otros países latinoamericanos, como Bolivia, Guatemala y Venezuela.

A partir de las estadísticas reportadas en las Evaluaciones Agropecuarias, se identificaron tendencias decrecientes en la producción en los departamentos que conforman el Eje Cafetero colombiano: Caldas, Quindío, Risaralda y Valle del Cauca; en Tolima y en el Eje cundiboyacense; en los demás departamentos se evidencia volatilidad con tendencias a la estabilidad.

Las estadísticas de las áreas cultivadas en Colombia, reportadas por la Federación, permiten identificar tendencias decrecientes en Caldas, Chocó, Cundinamarca, Quindío, Risaralda y Valle del Cauca; volatilidad con tendencia a la estabilidad en Boyacá y Norte de
Santander y tendencias crecientes en los demás departamentos evaluados.

Los factores relacionados con la variabilidad climática, el incremento del precio de producción, la escasez de mano de obra y la volatilidad del precio del café afectan la industria cafetera, y pueden ser causas estructurales de las tendencias decrecientes en la producción, encontradas en algunos países productores y en los departamentos tradicionalmente caficultores en Colombia.

La producción cafetera se ha afectado por la variabilidad climática, pero los impactos del cambio climático podrían ser más severos. En este sentido, se requiere avanzar en la modelación de cultivos y la evaluación de los factores que perjudican la productividad cafetera, de tal manera que permitan estimar posibles impactos por estos fenómenos y establecer acciones efectivas para la adaptación. En este sentido, se requiere conocer con mayor detalle las estadísticas de producción, en lo posible, del nivel municipal y veredal, y a escala mensual.

Se recomienda un análisis de las estrategias de adaptación a la variabilidad y cambio climático en los sistemas cafeteros de países latinoamericanos que presentan tendencias crecientes en la producción de café arábiga, como es el caso de Brasil, Honduras, Nicaragua y Perú. 


\section{REFERENCIAS}

Baker, P. (2010). Peak Coffee? London, UK: International Coffee Organization, ICO World Conference.

Banco Interamericano de Desarrollo \& Comisión Económica para América Latina y el Caribe. (2012). Valoración económica de daños y pérdidas. Ola invernal Colombia 2010-2011. Bogotá: BID-CEPAL.

Cadena G. (2005). Desarrollos científicos de CENICAFÉ en la última década. Revista de la Academia Colombiana de Ciencias, 29(110), 89-99.

CBI Ministry of Foreign Affairs (2016). Which trends offer opportunities on the European coffee market? Retrieved from https://www.cbi.eu/marketinformation/coffee/trends/

Corporación Colombiana de Investigación Agropecuaria - CORPOICA. (2011). Investigación sobre los efectos del cambio climático en la distribución altitudinal de insectos plaga del café y sus enemigos naturales en la zona cafetera de Colombia. Bogotá: CORPOICA.

Departamento Nacional de Planeación -DNP-. (2011). Documento CONPES 3700. Estrategia Institucional para la articulación de políticas y acciones en materia de cambio climático en Colombia. Bogotá: DNP.

Echavarría, J. J., Esguerra, P., McAllister, D. \& Robayo, C. F. (2015). Informe de la misión de estudios para la competitividad de la caficultura en Colombia. Resumen ejecutivo. Bogotá: Universidad del Rosario.

El Espectador (2016, 16 marzo). Fenómeno de El Niño ha afectado cerca de 1,5 millones de sacos de café. El Espectador. En: http://www.elespectador.com/ noticias/economia/fenomeno-de-el-nino-ha-afectado-cerca-de-15-millones-de-articulo-622565

El País (2013, 25 de feb.). Estos son los cinco males que vive el sector cafetero en Colombia. El País. Recuperado de http://www.elpais.com.co/elpais/ economia/noticias/estos-son-cinco-males-vive-sector-cafetero-colombia 
Apuntes CENES Volumen 36, Número 64

julio - diciembre 2017. Págs. 139-165

El Tiempo (2016 $6^{\mathrm{a}}, 2$ jun.). El fenómeno del Niño le costó al país 1,6 billones de pesos. El Tiempo. Recuperado de http://www.eltiempo.com/estilo-de-vida/ ciencia/impacto-del-fenomeno-del-nino-en-colombia-2016/16609985

El Tiempo (2016b, 16 marzo). El fenómeno del Niño (sic) dejará pérdidas por $\$ 0,5$ billones al café. El Tiempo. Recuperado de http://www.eltiempo. com/economia/sectores/perdida-de-cafeteros-por-el-fenomeno-de-el-nino/16538839

Esguerra, M. \& MCAllister, D. (2013). El mercado internacional del café verde. Su evolución reciente y sus perspectivas 2013-2020. Bogotá: Universidad del Rosario.

Federación Nacional de Cafeteros de Colombia -FNC-. (2011). Comportamiento de la industria cafetera colombiana 2011. Bogotá: FNC.

Federación Nacional de Cafeteros de Colombia -FNC-. (2012). Caficultura sostenible. Informe del gerente general. 2012. Bogotá: FNC.

Federación Nacional de Cafeteros de Colombia -FNC- (2013). Comportamiento de la industria cafetera colombiana 2012. Bogotá: FNC.

Federación Nacional de Cafeteros de Colombia -FNC-. (2014a). La política cafetera 2010-2014. Bogotá: FNC.

Federación Nacional de cafeteros de Colombia -FNC-. (2014b). ¿Cómo impactan El Niño y La Niña la producción de café en Colombia? Recuperado de http:/www.cafedecolombia.com/bb-fnc-es/index.php/comments /como impactan_el_nino_y_la_nina_la_produccion_de_cafe_de_colombia

Federación Nacional de Cafeteros de Colombia -FNC-. (2015a). Comportamiento de la industria cafetera colombiana 2014. Bogotá: FNC.

Federación Nacional de Cafeteros de Colombia. (2015b). Unidos por la rentabilidad del caficultor. En LXXXII Congreso Nacional de Cafeteros, celebrado en Bogotá.

Federación Nacional de Cafeteros de Colombia -FNC-. (2016a). Área cultivada con café total departamental. Recuperado de $\mathrm{http}: / / \mathrm{www}$. federaciondecafeteros.org/particulares/es/ quienes_somos/119_estadisticas_historicas/ 
Federación Nacional de Cafeteros de Colombia -FNC-. (2016b). Producción de café de Colombia fue de 944 mil (sic) sacos en marzo. Recuperado de http://www.cafedecolombia.com/cci-fnc-es/index.php/comments/produccion_de_cafe_de_colombia_fue_de_944_mil_sacos_en_marzo/

Federación Nacional de Cafeteros de Colombia -FNC-. (2016c). Volumen de exportaciones colombianas de café según destino anual. Recuperado de http://www.federaciondecafeteros.org/particulares/es/quienes_somos/119_estadisticas_historicas/

Hirsch, R.M., Slack, J.R. \& Smith, R.A. (1982). Techniques for Trend Assessment for Monthly Water Quality Data. Water Resources Research, 18, 107121. Retrieved from https://doi.org/10.1029/WR018i001p00107

International Coffee Organization -ICO-. (2016a). Exportable production by all exporting countries. London: ICO. Retrieved from http://www.ico.org/new_historical.asp?section=Statistics

International Coffee Organization -ICO-. (2016b). Total production by all exporting countries. London. Retrieved from http://www.ico.org/ new_historical.asp?section=Statistics

Jaramillo-Robledo, A. \& Arcila-Pulgarín, J. (2009). Variabilidad climática en la zona cafetera colombiana asociada al evento de La Niña y su efecto en la caficultura. Avances Técnicos Cenicafé, 389, 1-9.

Laderach, P., Lundy, M., Jarvis, A., Ramírez, J., Portilla, E. P., Schepp, K. \& Eitzinger, A. (2011). Predicted Impact of Climate Change on Coffee Supply Chains. In The Economic, Social and Political Elements of Climate Change (pp. 703-723). Berlin, Heidelberg: Springer. https://doi.org/10.1007/9783-642-14776-0 42

La República. (2014, 19 nov.). OIC prevé que la demanda global de café aumente 2,5\% anual hasta el 2020. La República. Recuperado de http://www.larepublica.co/oic-prev\%C3\%A9-que-la-demanda-global-de-caf\%C3\%A9aumente-25-anual-hasta-el-2020_193271

La República. (2015, 13 abr.). Cosecha cafetera necesita de 300.000 recolectores que no ha logrado atraer. La República. Recuperado de http:// www.larepu-blica.co/cosecha-cafetera-necesita-de-300000-recolectoresque-no-ha-lo-grado-atraer_242276 
Apuntes CENES Volumen 36, Número 64

julio - diciembre 2017. Págs. 139-165

Ministerio de Agricultura y Desarrollo Rural. (2014). Evaluaciones agropecuarias municipales 2013. Bogotá: Grupo de Estadísticas e Información Sectorial - Oficina Asesora de Planeación y Prospectiva - Ministerio de Agricultura y Desarrollo Rural.

NOAA. (2016). Cold \& Warm Episodes by Season. Retrieved from http://www.cpc.ncep.noaa.gov/products/analysis_monitoring/ensostuff/ ensoyears.shtml

Ocampo, O. (2011, enero-junio). El cambio climático y su impacto en el agro. Revista de Ingeniería, (33), 115-123.

Ocampo, O. (2016). Modelación hidrológica y agronómica de los efectos del cambio y la variabilidad climática en la producción cafetera de Caldas. Manizales: Universidad Nacional de Colombia sede Manizales.

Ocampo, O. (2017). Modelación hidrológica y agronómica de los efectos del cambio y la variabilidad climática en la producción cafetera de Caldas. Manizales: Universidad Nacional de Colombia sede Manizales.

Ocampo-López, O. L., Ovalle-Castiblanco, A. M., Arroyave-Díaz, A., Salazar-Ospina, K., Ramírez-Gómez, C. A. \& Oliveros-Tascón, C. E. (2017). Nuevo método estándar para la recolección selectiva de café. Ingeniería Investigación y Tecnología, 28(2), 127-137.

Ocampo, Vélez \& Londoño (2014). Análisis de vulnerabilidad hídrica de la cuenca del río Chinchiná. Manizales: Universidad Nacional de Colombia. Facultad de Ingeniería y Arquitectura.

Organización Internacional del Café -OIC-. (2014a). Anuario 2013-2014. Fortaleciendo el sector cafetero mundial mediante la cooperación internacio-nal. Londres: OIC. Recuperado de www.ico.org

Organización Internacional del Café -OIC-. (2014b). Comercio mundial del café (1963-2013) reseña de los mercados, retos y oportunidades con que se enfrenta el sector. Vol. ICC 111-5. London: OIC.

Organización Internacional del Café -OIC-. (2015). Anuario 2014 - 2015 Fortaleciendo el sector cafetero mundial mediante la cooperación internacional. Londres: OIC. Retrieved from http://www.ico.org/documents/cy201516/annual-review-2014-15-c.pdf 
Organización Internacional del Café -OIC-. (2016). Informe del mercado de café-febrero 2016. Aumenta el consumo mundial de café, pero los precios siguen bajos. Londres: OIC.

Portafolio (2015, 4 de jun.). Sector cafetero se enfrenta a un grave problema. ¿Cuál es? Portafolio. Recuperado de http://www.portafolio.co/ economia/finanzas/sector-cafetero-enfrenta-grave-problema-40414.

Poveda, G., Turbay, S., Vélez, J. J., Lucía, O., Cristina, E. \& Bedoya, M. (2014). ¡ No sé qué vamos a hacer con estos climas! Vulnerabilidad y adaptación a las variaciones climáticas extremas en la cuenca de la quebrada Los Cuervos, afluente del río Chinchiná, Colombia. Medellín: Universidad de Antioquia, Universidad Nacional de Colombia, IDRC, CIHT.

Quintero, L. \& Rosales, M. (2014). El mercado mundial del café: tendencias recientes, estructura y estrategias de competitividad. Visión Gerencial, 13(2), 291-307.

Ramírez, J., Salazar, M., Jarvis, A. \& Navarro, C. (2012). A way forward on adaptation to climate change in Colombian agriculture: perspectives towards 2050. Climatic Change, 115, 611-628. https://doi.org/10.1007/s10584012-0500-y

Salazar, K., Arroyave, A., Ovalle, A. M., Ocampo, O. L., Ramírez, C. \& Oliveros, C. E. (2016). Tiempos en la recolección manual tradicional de café. Ingeniería Industrial, 37(2), 114-126.

Sauchyn, D., Upegui, J.J, Masiokas, M., Ocampo, O., Cara, L. \& Villalba, R. (2016). Exposure of Rural Communities to Climate Variability and Change: Case Studies from Argentina, Colombia and Canada. In W. Leal Filjo, K. Adamson, R. M. Dunk, U. Alzeiteiro, S. Illingworth \& F. Alves (Eds.), Implementing Climate Change Adaptation in Cities and Communities. Integrating Strategies and Educational Approaches (pp. 23-38). Springer. http://doi.org/10.1007/978-3-319-28591-7

Schroth, G., Laderach, P., Dempewolf, J., Philpott, S., Haggar, J., Eakin, H., Castillejos, T., García-Moreno, J., Soto-Pinto, L., Hernández, R., Eitzinger, A. \& Ramírez-Villegas, J. (2009). Towards a climate change adaptation 
strategy for coffee communities and ecosystems in the Sierra Madre de Chiapas, Mexico. Mitig Adapt Strateg Glob Chang 14, 605-625. https:// doi.org/10.1007/s11027-009-9186-5

Superintendencia de Industria y Comercio. (2012). Estudios de mercado. Estudio sobre el sector del café en Colombia. Bogotá: Grupo de Estudios Económicos. SIC.

Tucker, C., Eakin, H. \& Castellanos, E. (2010). Perceptions of Risk and Adaptation: Coffee Producers, Market Shocks, and Extreme Weather in Central America and Mexico. Global Environmental Change, 20(1), 23-32. https://doi.org/10.1016/j.gloenvcha.2009.07.006

Turbay, S., Nates, B., Jaramillo, F. L., Vélez, J. J. \& Ocampo, O. L. (2014). Adaptación a la variabilidad climática entre los caficultores de las cuencas de los ríos Porce y Chinchiná, Colombia. Investigaciones Geográficas, Boletín del Instituto de Geografia, 0(85), 95-112. Recuperado de http://doi. org/10.14350/rig.42298 
